\title{
Métodos numéricos simplécticos de un paso
}

\section{Simple one-step numerical methods}

\author{
G. Gonzáles-Santos* \\ Escuela Superior de Física y Matemáticas \\ Instituto Politécnico Nacional - IPN, México
}

\begin{abstract}
Resumen. Los métodos numéricos geométrico son empleados para aproximar la solución de sistemas Hamiltonianos y conservar propiedades importantes de estos sistemas; como son la energía, las áreas y las simetrías, entre otras. En este artículo se presentan algunos métodos simplécticos de un paso (Runge-Kutta). Los métodos obtenidos han sido empleados en la solución de varias problemas de mecánica. Se incluyen, en este trabajo, algunos resultados del estudio numérico de vibraciones en una cuerda elástica vertical obtenidos por medio de métodos simplécticos.
\end{abstract}

Palabras Claves. Métodos numéricos, hamiltonianos, mecánica clásica, métodos simplécticos.

\begin{abstract}
Geometric numerical methods are used to approximate the solution of Hamiltonian systems and to preserve important properties of these systems; such as energy, areas and symmetries, among others. In this article some simple one-step methods (Runge-Kutta) are presented. The methods obtained have been used in the solution of several mechanical problems. Some results of the numerical study of vibrations in a vertical elastic cord obtained by means of simplistic methods are included in this work.
\end{abstract}

Keywords. Numerical methods, Hamiltonian, classical mechanics, simplistic methods.

Como citar. G. Gonzáles-Santos, "Métodos numéricos simplécticos de un paso", Jou. Cie. Ing., vol. 11, no 1, pp. 7-24, 2019. doi: https://doi.org/10.46571/JCI.2019.1.2

\section{Introducción}

Existe una amplia gama de métodos numéricos para resolver el problema de valores iniciales de ecuaciones diferenciales ordinarias,

$$
\begin{aligned}
\mathbf{y}^{\prime} & =\mathbf{f}(x, \mathbf{y}), \quad a<x<b \\
\mathbf{y}(a) & =\mathbf{y}_{a},
\end{aligned}
$$

donde $\mathbf{y}$ es un escalar o un vector e $\mathbf{y}_{a}$ es la condición inicial. Los métodos numéricos clásicos, como son, los métodos de Taylor, un paso, multipaso, extrapolación y predictor corrector, [1], no fueron desarrollados para que la diferencia finita asociada al método comparta propiedades con el problema de partida. Por ejemplo, considere el péndulo simple el cual es un sistema conservativo, es decir, la energía total (cinética más potencial) se mantiene constante en el tiempo. En la figura 1 se muestra un 
péndulo simple. La derivación del modelo del péndulo simple puede encontrar en diversos textos, por ejemplo, [2, p. 348],

$$
\ddot{\phi}(t)+\frac{g}{l} \operatorname{sen}(\phi)=0,
$$

donde $\phi(t)$ es el ángulo entre la vertical y el cable del péndulo, $g$ es la gravedad, y $l$ es la longitud del cable que sostiene la masa $m, \dot{\phi}$ denota la derivada temporal de $\phi$. El complemento de este modelo son las condiciones iniciales, $\phi(0)$ y $\dot{\phi}(0)$.

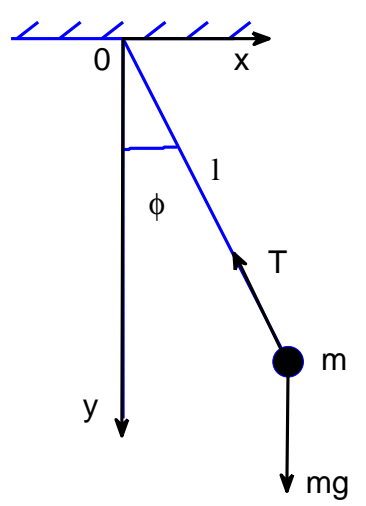

Figura 1: El péndulo simple

La energía cinética $K(t)$, potencial, $V(t)$ y total, $E(t)$ están dadas por la relaciones siguientes:

$$
\begin{aligned}
K(t) & =\frac{1}{2} m l^{2}(\dot{\phi})^{2}, \\
V(t) & =m g l(1-\cos (\phi)), \\
E(t) & =K(t)+V(t) .
\end{aligned}
$$

Las trayectorias del péndulo simple en el espacio fase para diferentes valores de energía se muestran en la figura 2. El círculo más interno corresponde a una energía de $E(t)=0,134[J]$. El resto de las curvas se obtienen a partir del nivel de energía de $0,5[J]$ hasta $4[J]$ con incrementos de $0,5[J]$.

Ahora se realiza el siguiente experimento numérico usando el método de Euler. Los parámetros del péndulo son los siguientes: $l=1[\mathrm{~m}], g=1\left[\mathrm{~m} / \mathrm{seg}^{2}\right]$ y $m=1[\mathrm{Kg}]$. El experimento consiste en elegir la trayectoria en el espacio fase con nivel de energía, $E(t)=0,134[J]$, y representarla por un conjunto de puntos, $N$, uniformemente espaciados. Estos puntos son las condiciones iniciales para el método de Euler, es decir, se van a resolver $N$ problemas con valores iniciales y las condiciones iniciales son cada punto de la curva en el espacio fase. Como el sistema es conservativo los puntos deben permanecer sobre la misma curva (solo pueden rotar).

En el experimento numérico se obtiene la evolución de las partículas y se grafican en el espacio fase cada $500[\mathrm{seg}]$. La figura 3 muestra los resultados obtenidos. La primera observación es que las partículas no se mantienen en su mismo nivel de energía, éstas ganan energía al avanzar el tiempo. Por lo tanto desde el punto de vista numérico, el sistema no es conservativo. La segunda observación es sobre el área que delimita la curva incial. Ésta no no se mantiene constante; aumenta al avanzar el tiempo. La tercera observación es sobre el tiempo de integración.

El experimento numérico se realizó con una longitud de paso constante, $h=0,0005$, por lo tanto se requiere de un millon de iteraciones del método de Euler para pasar de un círculo al siguiente. Por lo tanto es importante para un método numérico conservar ciertas propiedades del problema inicial cuando se estudia durante periodos largos de tiempo. La figura 4 muestra la evolución de la energía total del 


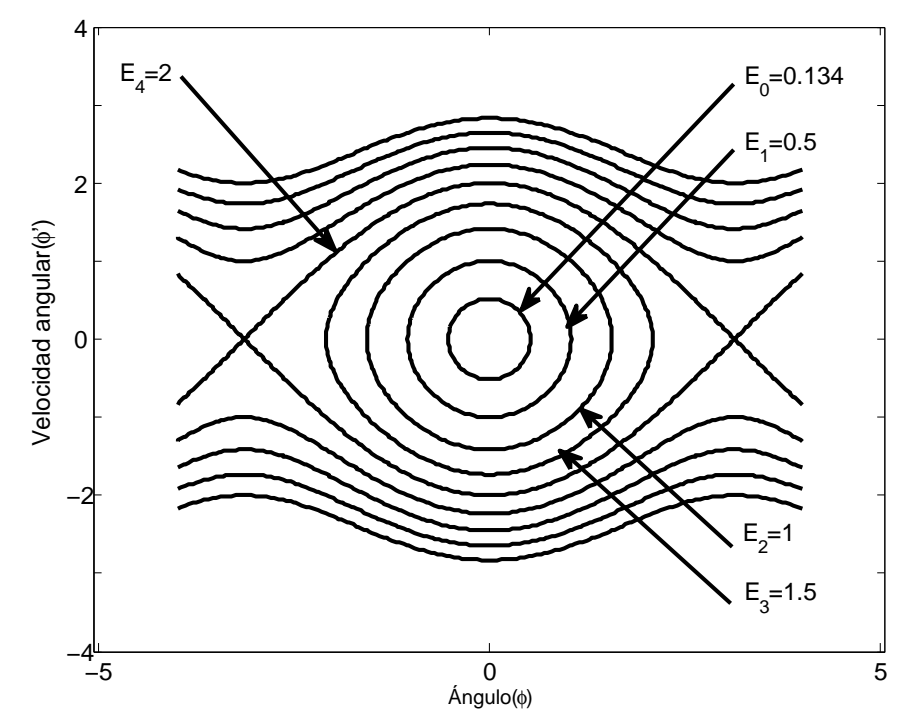

Figura 2: Espacio fase del péndulo simple para diferentes valores de energía.

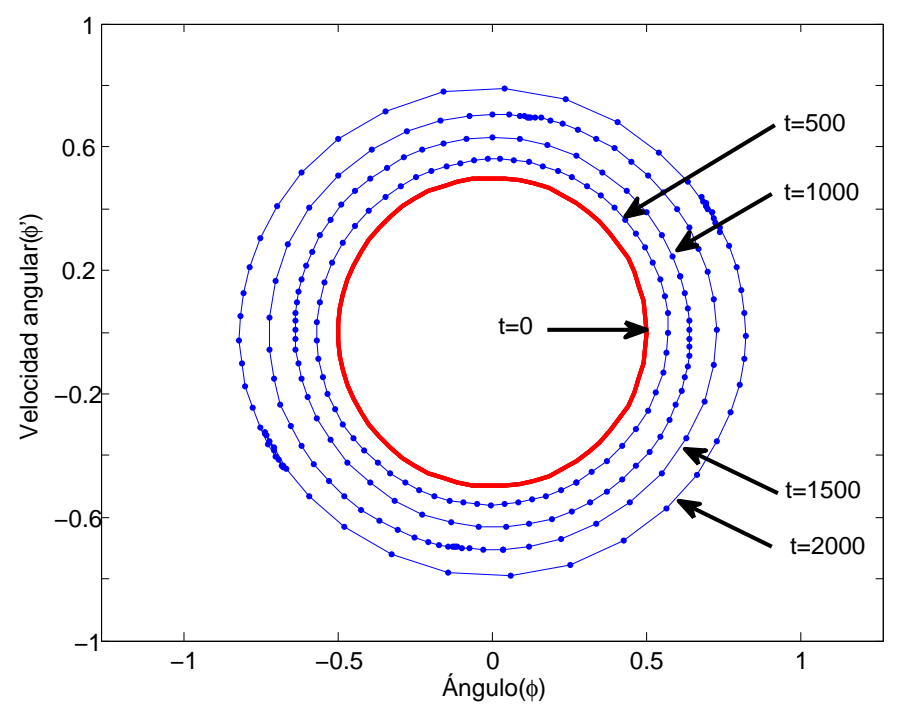

Figura 3: Ubicación, en el espacio fase, de los puntos que inicialmente $(t=0)$ se encuentran sobre la curva con energía $E=0,134$.

péndulo simple con condición inicial, $\phi(0)=\pi / 6$ y $\dot{\phi}(0)=0$. De acuerdo a los resultados numéricos el péndulo gana energía al avanzar el tiempo.

El comportamiento obtenido con el método de Euler también está presenta en la mayoría de los métodos clásicos. En áreas como la mecánica, existen propiedades importantes que deben considerarse, como son:

1. Conservación de áreas (volumenes) en el espacio fase.

2. Conservación de la energía total (Hamiltoniano).

3. Mantener la simétria. El Hamiltoniano es una función simétrica respecto al momento, $H(\mathbf{q}, \mathbf{p})=$ $H(\mathbf{q},-\mathbf{p})$.

4. Conservación de las primeras integrales (momento lineal total, momento angular lineal, etc.) 


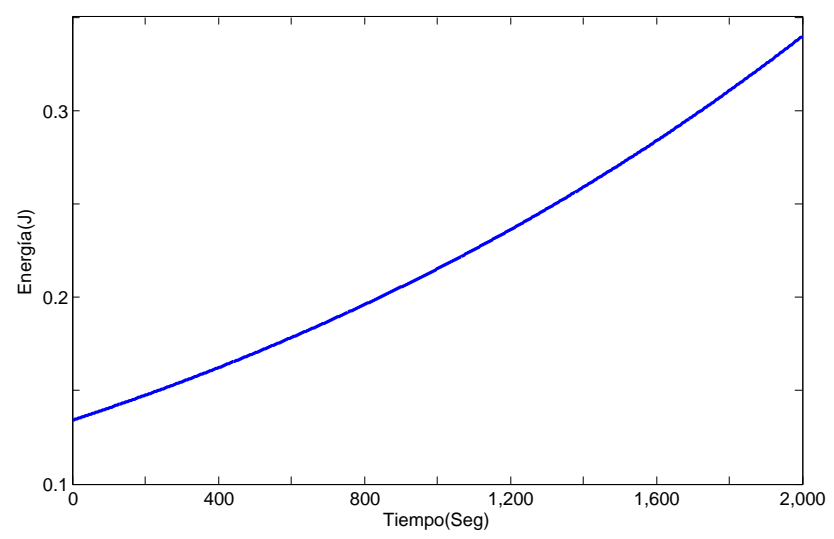

Figura 4: Evolución de la energía del péndulo simple con condiciones iniciales, $\phi(0)=\pi / 6$ y $\dot{\phi}(0)=0$. En este caso, $E(t)=0,134[J]$.

El presente trabajo está dedicado al estudio de métodos numéricos que preservan una o varias estructuras de ecuaciones diferenciales ordinarias Hamiltonianas, es decir, sistemas de la forma,

$$
\begin{aligned}
\dot{\mathbf{q}} & =\nabla_{\mathbf{p}} H(\mathbf{p}, \mathbf{q}) \\
\dot{\mathbf{p}} & =-\nabla_{\mathbf{q}} H(\mathbf{p}, \mathbf{q})
\end{aligned}
$$

donde $H(\mathbf{p}, \mathbf{q})$ es el Hamiltonianao y es una función, $H:^{d} \times^{d} \rightarrow$ y $d$ es el número grados de libertad. En aplicaciones el Hamiltoniano tiene la forma,

$$
H(\mathbf{p}, \mathbf{q})=\frac{1}{2} \mathbf{p}^{t} M^{-1} \mathbf{p}+U(\mathbf{q})
$$

con una matriz $M$ definida positiva y $U(\mathbf{q})$ la función potencial. En este situación el Hamiltoniano representa la energía del sistema. Sistemas Hamiltonianos de ecuaciones diferenciales ordinarias se encuentran en diversas aplicaciones (mecánica, problema de $n$ cuerpos, astrofísica, etc.)

\section{El problema de $N$ cuerpos}

Un tema central en los cursos de mécanica clásica es la segunda Ley de Newton la cual se aplica, entre otras cosas, al estudio de sistemas formado por uno o varios puntos masa (partículas). La fuerza actuante sobre una partícula proviene de dos fuentes; la primera está formada por las partículas vecinas y segunda por un campo externo, como puede ser la gravedad.

\subsection{Formulación Newtoniana}

Esta formulación produce un sistema de de ecuaciones diferenciales de segundo orden con condiciones iniciales. Formalmente este problema, conocido como el problema de los $N$ cuerpos, puede pantearse como sigue: dados $N$ cuerpos (partículas) $P_{1}, P_{2}, \cdots, P_{N}$ con masas $m_{1}, m_{2}, \cdots, m_{N}$, las cuales son independientes de la posición, velocidad y del tiempo. Las posiciones de las partículas, en el tiempo $t$, se denotan por los vectores, $\mathbf{r}_{1}(t), \mathbf{r}_{2}(t), \cdots, \mathbf{r}_{N}(t)$. La dimensión de estos vectores depende del problema. Sea $\mathbf{F}_{i, j}$ la fuerza que ejerce la partícula $j$-ésima sobre la partícula $i$-ésima. La fuerza actúa en la dirección del vector $\mathbf{r}_{i, j}=\mathbf{r}_{i}-\mathbf{r}_{j}$, además se asume que $F_{i, j}=F_{j, i}$. La fuerza que ejerce un campo externo sobre la $i$-ésima partícula se denota por $\mathbf{f}_{i}$. Por lo tanto la ecuación de movimiento queda establecida al aplicar la segunda ley de Newton al sistema de partículas, 


$$
\begin{aligned}
m_{i} \frac{d^{2} \mathbf{r}_{i}}{d t^{2}} & =\sum_{j=1, j \neq i}^{N} \mathbf{F}_{i, j}+\mathbf{f}_{i}, \quad i=1,2, \cdots, N \\
\mathbf{r}_{i}(0) & =\mathbf{r}_{i}^{0} \\
\mathbf{r}_{i}^{\prime}(0) & =\mathbf{v}_{i}^{0}
\end{aligned}
$$

El tamaño del sistema depende de la dimensión del problema; en el plano se tienen $2 N$ ecuaciones diferenciales ordinarias de segundo orden y en el espacio $3 N$.

Ejemplo. Una caso interesante en el problema de $N$ cuerpos es cuando el sistema es conservativo, es decir, la energía del sistema se mantiene constante. En este caso la fuerza se obtiene como menos el gradiente de una función potencial,

$$
F=-\nabla V\left(\mathbf{r}_{1}, \mathbf{r}_{2}, \cdots, \mathbf{r}_{N}\right)
$$

la cual depende solamente de la posición de las partículas. Un sistema de este tipo se obtiene cuando se estudia una cuerda elástica, la cual es representa por $N$ partículas, $P_{1}, P_{2}, \cdots, P_{N}$ con masas $m_{1}$, $m_{2}, \cdots, m_{N}$ respectivamente. La masa $i$-ésima está unida a las masas $i+1$ e $i+1$ por medio de un resorte, excepto cuando la masa está en el extremo de la cuerda. El número de resortes en el sistema, $M$, depende de las condiciones de frontera; si ambos extremos están fijos $M=N+1$ y $M=N$ si alguno de los extremos está libre. La longitud inicial de cada resorte es $1_{1}, 1_{2}, \ldots, l_{M}$ respectivamente, y $k_{l}, k_{2}$, $\ldots, k_{M}$ son los correspondientes coeficientes de rigidez, además se considera que los resortes carecen de masa. En este ejemplo se considera una cuerda vertical fija en el extremo superior y libre en el otro $(N=M)$. Se asume que las partículas se deslizan sin fricción en el plano $x-y$, ver figura 5 .

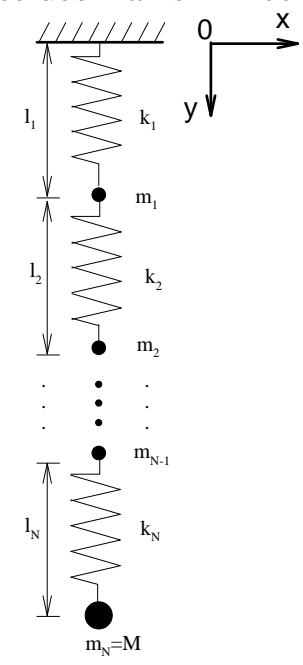

Figura 5: Modelo discreto de una cuerda elástica

Frecuentemente el movimiento de la cuerda se analiza a partir de su posición de equilibrio. Esta se obtiene al resolver el sistema algebraico obtenido de (3) al hacer cero el lado izquierdo,

donde

$$
K y^{e q}=-(T \mathbf{l}+g M \mathbf{e})
$$

$$
K=\left(\begin{array}{ccccc}
-\left(k_{1}+k_{2}\right) & k_{2} & & & \\
k_{2} & -\left(k_{2}+k_{3}\right) & k_{3} & & \\
& k_{3} & \ddots & \ddots & \\
& & \ddots & & k_{N} \\
& & & k_{N} & -k_{N}
\end{array}\right) \text {, }
$$




$$
T=\left(\begin{array}{ccccc}
k_{1} & -k_{2} & & & \\
& k_{2} & -k_{3} & & \\
& & \ddots & \ddots & \\
& & & k_{N-1} & -k_{N} \\
& & & & k_{N}
\end{array}\right),
$$

$M=\operatorname{diag}\left(m_{1}, \cdots, m_{N}\right), \mathbf{l}=\left(l_{1}, l_{2}, \cdots, l_{N}\right)^{t}$ y $\mathbf{e}=(1,1, \cdots, 1)^{t}$. Los vectores posición de la partículas en equilibrio son

$$
\mathbf{r}_{i}^{e q}=\left(0, y_{i}^{e q}\right), \quad i=1,2, \cdots, N
$$

La energía cinética y potencial de la cuerda elástica son respectivamente,

$$
\begin{aligned}
K & =\frac{1}{2} \sum_{i=1}^{N} m_{i} \dot{r}_{i}^{2}, \\
V & =\frac{1}{2} \sum_{i=1}^{N} k_{i}\left(\left\|\mathbf{r}_{i}-\mathbf{r}_{i-1}\right\|-l_{i}\right)^{2}-g \sum_{i=1}^{N} m_{i} y_{i},
\end{aligned}
$$

con $\mathbf{r}_{0}=\mathbf{0}, \mathbf{r}_{i}=\left(x_{i}, y_{i}\right)^{t}$ y $r_{i}=\left\|\mathbf{r}_{i}\right\|=\sqrt{x_{i}^{2}+y_{i}^{2}}$. En este ejemplo, el término de la fuerza de la ecuación de movimiento, (3), es el negativo del gradiente del potencial $(V)$. La $i$-ésima ecuación de movimiento se obtiene a partir de las derivadas parciales de $V$ con respecto a $x_{i}$ e $y_{i}$,

$$
\begin{aligned}
\frac{\partial V}{\partial x_{i}}= & -k_{i+1}\left[\left(r_{i, i+1}-l_{i+1}\right) \frac{\left(x_{i}-x_{i+1}\right)}{r_{i, i+1}}\right]+ \\
& k_{i}\left[\left(r_{i, i-1}-l_{i}\right) \frac{\left(x_{i}-x_{i-1}\right)}{r_{i, i-1}}\right] \\
\frac{\partial V}{\partial y_{i}}= & -k_{i+1}\left[\left(r_{i, i+1}-l_{i+1}\right) \frac{\left(y_{i}-y_{i+1}\right)}{r_{i, i+1}}\right]+ \\
& k_{i}\left[\left(r_{i, i-1}-l_{i}\right) \frac{\left(y_{i}-y_{i-1}\right)}{r_{i, i-1}}\right]-g m_{i} .
\end{aligned}
$$

La ecuación de movimiento de la partícula $i$-ésima resulta,

$$
\begin{aligned}
m_{i} \frac{d^{2} \mathbf{r}_{i}}{d t^{2}}= & -\left[\frac{\partial V}{\partial x_{i}}, \frac{\partial V}{\partial y_{i}}\right] \\
= & k_{i+1}\left[\left(r_{i, i+1}-l_{i+1}\right) \frac{\left(\mathbf{r}_{i, i+1}\right)}{r_{i, i+1}}\right]- \\
& k_{i}\left[\left(r_{i, i-1}-l_{i}\right) \frac{\left(\mathbf{r}_{i, i-1}\right)}{r_{i, i-1}}\right]+g m_{i} \hat{e}_{j}
\end{aligned}
$$

donde $\hat{e}_{j}$ es el vector unitario en la dirección $y$.

\subsection{Formulación Hamiltoniana}

Otra manera de formular el problema de $N$ cuerpos es emplear su formulación Hamiltoniana, la cual produce un sistema de ecuaciones diferenciales de primer orden. Para este propósito se introducen dos 
nuevos grupos de variables; Las coordenadas generalizadas, $\mathbf{q}(t)$. Si se asume que el problema está en plano entonces

$$
q_{2 i-1}=x_{i}, \quad q_{2 i}=y_{i}, \quad i=1,2, \ldots, N .
$$

La otra variable son los momentos generalizados o momentos conjugados, $\mathbf{p}(t)$,

$$
\mathbf{p}=M \dot{\mathbf{q}},
$$

donde $M \in \mathbb{R}^{2 N} \times \mathbb{R}^{2 N}$ es una matriz diagonal que contiene las masas repetidas dos veces, es decir,

$$
M_{2 i-1,2 i-1}=m_{i} \quad M_{2 i, 2 i}=m_{i}, \quad i=1,2, \ldots, N .
$$

La segunda ley de Newton, (3), se puede escribir de la manera siguiente:

$$
\begin{aligned}
\dot{\mathbf{q}} & =M^{-1} \mathbf{p}, \\
\dot{\mathbf{p}} & =-\nabla_{\mathbf{q}} V(\mathbf{q}) .
\end{aligned}
$$

Estas ecuaciones forman un sistema Hamiltoniano de ecuaciones diferenciales ordinarias con un Hamiltoniano o energía total igual a,

$$
H(\mathbf{p}, \mathbf{q})=\frac{1}{2} \mathbf{p}^{t} M^{-1} \mathbf{p}+V(\mathbf{q}) .
$$

Ejemplo. La forma Hamiltoniana del modelo discreto de la cuerda elástica resulta se obtiene directamente de la expresión de la energía,

$$
\begin{aligned}
H(\mathbf{p}, \mathbf{q})= & \frac{1}{2} \mathbf{p}^{t} M^{-1} \mathbf{p}+ \\
& \frac{1}{2} \sum_{i=1}^{N} k_{i}\left(\left\|\mathbf{r}_{i}-\mathbf{r}_{i-1}\right\|-l_{i}\right)^{2}- \\
& g \sum_{i=1}^{N} m_{i} q_{2 i},
\end{aligned}
$$

donde $\mathbf{r}_{i}=\left(q_{2 i-1}, q_{2 i}\right), i=1,2, \ldots, N$. La formulación Hamiltoniana del modelo discreto de la cuerda elástica vertical resulta,

$$
\begin{aligned}
\dot{q}_{2 i-1}= & p_{2 i-1} / m_{i}, \\
\dot{q}_{2 i}= & p_{2 i} / m_{i}, \\
\dot{p}_{2 i-1}= & -k_{i}\left(\left\|\mathbf{r}_{i}-\mathbf{r}_{i-1}\right\|-l_{i}\right) \frac{q_{2 i-1}}{\left\|\mathbf{r}_{i}-\mathbf{r}_{i-1}\right\|}+ \\
& k_{i+1}\left(\left\|\mathbf{r}_{i+1}-\mathbf{r}_{i}\right\|-l_{i+1}\right) \frac{q_{2 i-1}}{\left\|\mathbf{r}_{i+1}-\mathbf{r}_{i}\right\|}, \\
\dot{p}_{2 i}= & -k_{i}\left(\left\|\mathbf{r}_{i}-\mathbf{r}_{i-1}\right\|-l_{i}\right) \frac{q_{2 i}}{\left\|\mathbf{r}_{i}-\mathbf{r}_{i-1}\right\|}+ \\
& k_{i+1}\left(\left\|\mathbf{r}_{i+1}-\mathbf{r}_{i}\right\|-l_{i+1}\right) \frac{q_{2 i}}{\left\|\mathbf{r}_{i+1}-\mathbf{r}_{i}\right\|}+ \\
& m_{i} g, \quad i=1,2, \ldots, N .
\end{aligned}
$$

La formulación Hamiltoniana (5) se puede obtener a partir del Hamiltoniano de la manera siguiente: 


$$
\begin{aligned}
\dot{\mathbf{q}} & =\nabla_{\mathbf{p}} H(\mathbf{p}, \mathbf{q}), \\
\dot{\mathbf{p}} & =-\nabla_{\mathbf{q}} H(\mathbf{p}, \mathbf{q}) .
\end{aligned}
$$

Esta formulación se puede extender a una función suave $H(\mathbf{q}, \mathbf{p}): \mathbb{R}^{d} \times \mathbb{R}^{d} \rightarrow \mathbb{R}$. El espacio (espacio fase), $\mathbb{R}^{d} \times \mathbb{R}^{d}$, tiene dimensión $2 d$, la ecuación $(8)$ recibe el nombre de ecuación Hamiltoniana canónica de movimiento. Si se define $\mathbf{z}=(\mathbf{p}, \mathbf{q})^{t}$ entonces la ecuación anterior puede escribirse de manera compacta como,

$$
\dot{\mathbf{z}}=J \nabla_{\mathbf{z}} H(\mathbf{z}),
$$

donde

$$
J=\left(\begin{array}{cc}
0 & I \\
-I & 0
\end{array}\right)
$$

donde $I$ es la matriz identidad de dimensión $d$.

\section{Simplecticidad del flujo fase}

En esta sección se presentan algunas propiedades importantes de los sistemas Hamiltonianos, las cuales son una consecuencia de la estructura de estos sistemas. Por simplicidad se asume que el Hamiltoniano no depende del tiempo, es decir, $H=H(\mathbf{q}, \mathbf{p})=H(\mathbf{z})$ con $\mathbf{z}$ en el espacio fase, $\Omega \subseteq^{d} \times^{d}$. Las propiedades de los sistemas Hamiltonianos se expresan en términos del flujo fase del sistema, [3, p. 68]. Sea $\mathbf{z}^{\prime}=f(\mathbf{z}), \mathbf{z}(0)=\mathbf{z}_{0}$ un sistema Hamiltoniano y para cada $t$ se define el mapeo $\phi_{t}: \mathbf{z}_{0} \in \Omega \rightarrow \mathbf{z}(t) \in \Omega$, es decir, a cada condición inicial, $\mathbf{z}_{0} \in \Omega$ de se le asigna la solución del sistema en el tiempo $t, \phi_{t}\left(\mathbf{z}_{0}\right)=\mathbf{z}(t) \in \Omega$. La existencia y unicidad de la solución del sistema determinan el intervalo de tiempo donde $\phi_{t}$ existe. Para cada valor de $t$ el operador $\phi_{t}$ define un mapeo del espacio $\Omega$ en si mismo. Algunas propiedades del flujo fase de los sistemas Hamiltonianos son las siguientes, [4], [5], [4],

1. $\left\{\phi_{t}\right\}$ con la operación composición forma un grupo.

- Cerradura. $\phi_{t} \circ \phi_{s}=\phi_{t}\left(\phi_{s}\left(\mathbf{z}_{0}\right)\right)=\phi_{t}\left(\mathbf{z}_{s}\right)=\mathbf{z}_{t+s}=\phi_{t+s}$.

- Inverso. $\phi_{-t} \circ \phi_{t}=\phi_{0}=$ identidad.

- Asociatividad. $\phi_{r} \circ\left(\phi_{s} \circ \phi_{t}\right)=\phi_{r+s+t}=\left(\phi_{r} \circ \phi_{s}\right) \circ \phi_{t}$

2. El Hamiltoniano $H(\mathbf{q}, \mathbf{p})=H(\mathbf{z})$ es constante a lo largo de las soluciones del sistema Hamiltoniano. Esto quiere decir que la energía total se mantiene constante a lo largo de las soluciones.

$$
\dot{H}(\mathbf{q}(t), \mathbf{p}(t))=\nabla_{\mathbf{q}} \dot{\mathbf{q}}+\nabla_{\mathbf{p}} \dot{\mathbf{p}}=0 .
$$

3. El flujo fase $\phi_{t}$ del sistema Hamiltoniano es una transformación simpléctica. Para un $t$ fijo $\phi_{t}$ define una tranformación de $\Omega$ en si mismo,

$$
\hat{\mathbf{z}}=\psi(\mathbf{z}), \quad \forall \mathbf{z} \in \Omega .
$$

donde $\hat{\mathbf{z}}=\phi_{t}(\mathbf{z})$. La tranformación $\psi$ es simpléctica si satisface la condición siguiente,

$$
\left[\frac{\partial \hat{\mathbf{z}}}{\partial \mathbf{z}}\right]^{t} J\left[\frac{\partial \hat{\mathbf{z}}}{\partial \mathbf{z}}\right]=J
$$

donde $J$ es la matriz $(10)$ y

$$
\frac{\partial \hat{\mathbf{z}}}{\partial \mathbf{z}}=\left(\begin{array}{cc}
\frac{\partial \hat{\mathbf{q}}}{\partial \mathbf{q}} & \frac{\partial \hat{\mathbf{q}}}{\partial \mathbf{p}} \\
\frac{\partial \hat{\mathbf{p}}}{\partial \mathbf{q}} & \frac{\partial \hat{\mathbf{p}}}{\partial \mathbf{p}}
\end{array}\right) .
$$


De (11) se obtiene directamente que,

$$
\frac{\partial \hat{\mathbf{q}}}{\partial \mathbf{q}} \frac{\partial \hat{\mathbf{p}}}{\partial \mathbf{p}}-\frac{\partial \hat{\mathbf{q}}}{\partial \mathbf{p}} \frac{\partial \hat{\mathbf{p}}}{\partial \mathbf{q}}=I, \quad \forall(\mathbf{q}, \mathbf{p}) \in \Omega
$$

Esto significa que la transformación $\psi$ preserva áreas (volumenes) y orientación. Este resultado es el teorema de Liouville, [3, p. 69].

4. Si $H(\mathbf{q},-\mathbf{p})=H(\mathbf{q}, \mathbf{p})$ el flujo fase $\phi_{t}$ es $\rho$-reversible con respecto a la reflexión $\rho(\mathbf{q}, \mathbf{p})=$ $(\mathbf{q},-\mathbf{p})$, si satisface,

$$
\left(\rho \circ \phi_{t}\right)(\mathbf{z})=\left(\phi_{t}^{-1} \circ \rho\right)(\mathbf{z}), \quad \forall t \quad \forall \mathbf{z}
$$

\section{Métodos numéricos}

Los métodos numéricos estudiados en esta sección pueden emplearse indistintamente para la formulación Newtoniana o Hamiltoniana de un problema. Los algoritmos serán desarrollados para sistemas Hamiltonianos. La sección inicia con una lista de propiedades geométricas importantes que deben tenerse en cuenta al analizar un método para resolver (8). Los métodos desarrollados hasta el momento, para aproximar la solución de sistemas Hamiltonianos, cumplen una o varias propiedades de las listadas a continuación. Se denota con $\Phi_{h}\left(\mathbf{q}_{0}, \mathbf{p}_{0}\right)=\Phi_{h}\left(\mathbf{z}_{0}\right)$ la función de flujo de método numérico. Por medio de la composición de esta función,

$$
\left(\mathbf{q}_{n h}, \mathbf{p}_{n h}\right)=\Phi_{h} \circ \Phi_{h} \circ \cdots \circ \Phi_{h}\left(\mathbf{q}_{0}, \mathbf{p}_{0}\right),
$$

es posible aproximar la solución en tiempos posteriores. Algunas de las propiedades geométricas son las siguientes, [4, p. 69]:

1. Simétria. El método numérico es simétrico si satisface la relación siguiente,

$$
\Phi_{-h} \circ \Phi_{h}=\text { Identidad }
$$

2. Conservación de energía. El método conserva la energía si a lo largo de la solución de (8) se cumple que

$$
H\left(\mathbf{q}_{n}, \mathbf{p}_{n}\right)=\text { constante }
$$

3. Simplecticidad. Un métodos es simpléctico si satisface la siguiente relación,

$$
\Phi^{\prime}(\mathbf{p}, \mathbf{q})^{t} J \Phi^{\prime}(\mathbf{p}, \mathbf{q})=J,
$$

donde $\Phi(\mathbf{p}, \mathbf{q})=\nabla \Phi_{h} / \partial(\mathbf{q}, \mathbf{p})$ y $J$ es la matriz definida en 10$)$.

4. Reversibilidad. Un método numérico para aproximar la solución de 8 es $\rho$-reversible si, para un Hamiltoniano simétrico respecto a los momentos, $H(\mathbf{q}, \mathbf{p})=H(\mathbf{q},-\mathbf{p})$ se cumple

$$
\left(\rho \circ \Phi_{h}\right)(\mathbf{q}, \mathbf{p})=\left(\Phi_{-h} \circ \rho\right)(\mathbf{q}, \mathbf{p})
$$

Cuando un método numérico para aproximar la solución de un sistema Hamiltoniano cumple una o varias de las propiedades enunciadas se dice que el método es geométrico.

\subsection{Métodos de Runge-Kutta}

Los métodos implícitos de Runge-Kutta son una herramienta importante para el tratamiento de sistemas Hamiltonianos. Entre 1988 y 1989 fue demostrado por tres autores independientemente; Suris, [6], Lasagni, [7] y Sanz-Serna, [8], las condiciones bajo las cuales un método implícito de Runge-Kutta y de Runge-Kutta-Nystrom puede ser simplécticos. La forma general de un método de Runge-Kutta implícito de $m$ pasos para el problema de valores inciales,

$$
\mathbf{y}^{\prime}=f(x, \mathbf{y}), \mathbf{y}(a)=\mathbf{y}_{a}, \quad a \leq x \leq b,
$$


es la siguiente,

$$
\mathbf{y}_{k+1}=\mathbf{y}_{k}+h \sum_{i=1}^{m} w_{i} \mathbf{f}\left(x_{k}+\alpha_{i} h, \mathbf{Y}_{i}\right)
$$

con

$$
\begin{aligned}
\mathbf{Y}_{1} & =\mathbf{y}_{k}+h \sum_{j=1}^{m} \beta_{1, j} \mathbf{f}\left(x_{k}+\alpha_{j}, \mathbf{Y}_{j}\right) \\
\mathbf{Y}_{2} & =\mathbf{y}_{k}+h \sum_{j=1}^{m} \beta_{2, j} \mathbf{f}\left(x_{k}+\alpha_{j}, \mathbf{Y}_{j}\right) \\
\cdots & =\cdots \\
\mathbf{Y}_{m} & =\mathbf{y}_{k}+h \sum_{j=1}^{m} \beta_{m, j} \mathbf{f}\left(x_{k}+\alpha_{j}, \mathbf{Y}_{j}\right) .
\end{aligned}
$$

donde

$$
\alpha_{i}=\sum_{j=1}^{m} \beta_{i, j}, \quad i=1,2, \ldots, m .
$$

Una manera concisa de representar el método por por medio de la tabla de Butcher,

$$
\begin{array}{c|cccl}
\alpha_{1} & \beta_{1,1} & \beta_{1,2} & \cdots & \beta_{1, m} \\
\alpha_{2} & \beta_{2,1} & \beta_{2,2} & \cdots & \beta_{2, m} \\
\alpha_{3} & \beta_{3,1} & \beta_{3,2} & \cdots & \beta_{3, m} \\
\cdots & \cdots & \cdots & \cdots & \cdots \\
\alpha_{m} & \beta_{m, 1} & \beta_{m, 2} & \cdots & \beta_{m, m} \\
\hline & w_{1} & w_{2} & \cdots & w_{m}
\end{array}
$$

Para obtener la aproximación a la solución de (12), en cada paso de integración, hay que resolver el sistema algebraico no lineal (14) de $m * N$ ecuaciones.

Las condiciones para que un método de Runge-Kutta implícito sea simpléctico quedan establecidas en el teorema siguiente, [5, p. 72]:

Teorema. Si los coeficientes del método (13) 14) satisfacen las relaciones siguientes

$$
w_{i} \beta_{i, j}+w_{j} \beta_{j, i}=w_{i} w_{j}, \quad i, j=1,2, \ldots, m .
$$

Entonces el método es simpléctico.

Demostración. [5, p. 72].

Por ejemplo, el método de Runge-Kutta de punto medio implícito,

$$
\begin{array}{c|c}
1 / 2 & 1 / 2 \\
\hline & 1
\end{array}
$$

satisface las condiciones del teorema anterior por lo tanto es simpléctico. En este caso secillo se puede demostrar directamentente que el método anterior aplicado al sistema Hamiltoniano,

$$
\begin{aligned}
\dot{\mathbf{q}} & =\mathbf{p} \\
\dot{\mathbf{p}} & =\mathbf{f}(\mathbf{q})
\end{aligned}
$$

es simpléctico. El método de medio punto aplicado a sistema anterior resulta,

$$
\begin{aligned}
\mathbf{q}_{k+1} & =\mathbf{q}_{k}+h \mathbf{p}\left(t_{k}+h / 2\right) \\
\mathbf{p}_{k+1} & =\mathbf{p}_{k}+h \mathbf{f}\left(\mathbf{Q}_{1}\right) \\
\mathbf{Q}_{1} & =\mathbf{q}_{k}+\frac{h}{2} \mathbf{f}\left(\mathbf{Q}_{1}\right) .
\end{aligned}
$$


Multiplicando (15) por 2 y restando de (15b) se obtiene el valor de $Q_{1}$,

$$
\begin{aligned}
\mathbf{q}_{k+1} & =\mathbf{q}_{k}+h \mathbf{p}\left(t_{k}+h / 2\right) \\
\mathbf{p}_{k+1} & =\mathbf{p}_{k}+h \mathbf{f}\left(\mathbf{Q}_{1}\right) \\
\mathbf{Q}_{1} & =\frac{1}{2}\left(\mathbf{p}_{k}+\mathbf{p}_{k+1}\right)
\end{aligned}
$$

Sustituyendo $16 \mathrm{c}$ en $16 \mathrm{~b}$ y usando Taylor para aproximar $\mathbf{p}\left(t_{k}+h / 2\right)$ a segundo orden para mantener el orden del método de medio punto (segundo orden) se obtiene el método,

$$
\begin{aligned}
\mathbf{q}_{k+1} & =\mathbf{q}_{k}+\frac{h}{2}\left(\mathbf{p}_{k}+\mathbf{p}_{k+1}\right) \\
\mathbf{p}_{k+1} & =\mathbf{p}_{k}+h \mathbf{f}\left(\frac{1}{2}\left(\mathbf{p}_{k}+\mathbf{p}_{k+1}\right)\right) .
\end{aligned}
$$

El flujo fase de este método es

$$
\left(\begin{array}{l}
\mathbf{q}_{1} \\
\mathbf{p}_{1}
\end{array}\right) \Phi_{h}\left(\begin{array}{c}
\mathbf{q}_{0} \\
\mathbf{p}_{0}
\end{array}\right)=\left(\begin{array}{c}
\mathbf{q}_{0}+\frac{h}{2}\left(\mathbf{p}_{0}+\mathbf{p}_{1}\right. \\
\mathbf{p}_{0}+h \mathbf{f}\left(\frac{1}{2}\left(\mathbf{p}_{0}+\mathbf{p}_{1}\right)\right)
\end{array}\right) .
$$

Para verificar la simplécticidad del método es necesario calcular

$$
\left(\begin{array}{cc}
\frac{\partial \mathbf{q}_{1}}{\partial \mathbf{q}_{0}} & \frac{\partial \mathbf{q}_{1}}{\partial \mathbf{p}_{0}} \\
\frac{\partial \mathbf{1}_{1}}{\partial \mathbf{q}_{0}} & \frac{\partial \mathbf{q}_{1}}{\partial \mathbf{p}_{0}}
\end{array}\right)^{t} J\left(\begin{array}{cc}
\frac{\partial \mathbf{q}_{1}}{\partial \mathbf{q}_{0}} & \frac{\partial \mathbf{q}_{1}}{\partial \mathbf{p}_{0}} \\
\frac{\partial \mathbf{1}_{1}}{\partial \mathbf{q}_{0}} & \frac{\partial \mathbf{q}_{1}}{\partial \mathbf{p}_{0}}
\end{array}\right)
$$

donde

$$
J=\left(\begin{array}{cc}
0 & I_{N} \\
-I_{N} & 0
\end{array}\right)
$$

es una matriz $2 N \times 2 N$ con $I_{N}$ la matriz identidad de tamaño $N$. De la evaluación de esta expresión resulta,

$$
\left(\begin{array}{cc}
0 & I-\frac{1}{4} h^{2} \frac{\partial \mathbf{f}}{\partial \mathbf{q}_{0}} \\
-I+\frac{1}{4} h^{2} \frac{\partial \mathbf{f}}{\partial \mathbf{q}_{0}} & 0
\end{array}\right) .
$$

Por lo tanto si la longitud de paso, $h$, es pequeño entonces el método implícito de medio punto es simpléctico.

\subsection{Métodos de Runge-Kutta particionados(RKP)}

En estos métodos se emplean dos métodos de Runge-Kutta, uno de ellos se emplea para aproximar la solución de una parte del vector solución de un sistema de ecuaciones diferenciales ordinarias y el resto de la solución se aproxima con el otro método. Estos métodos pueden emplearse, por ejemplo, para aproximar las componentes de la solución no rígidas con un método y las rígidas con otro. Un método de Runge-Kutta particionado tiene asociadas dos tablas de Butcher,

\begin{tabular}{c|cccl}
$\alpha_{1}$ & $\beta_{1,1}$ & $\beta_{1,2}$ & $\cdots$ & $\beta_{1, m}$ \\
$\alpha_{2}$ & $\beta_{2,1}$ & $\beta_{2,2}$ & $\cdots$ & $\beta_{2, m}$ \\
$\alpha_{3}$ & $\beta_{3,1}$ & $\beta_{3,2}$ & $\cdots$ & $\beta_{3, m}$ \\
$\cdots$ & $\cdots$ & $\cdots$ & $\cdots$ & $\cdots$ \\
$\alpha_{m}$ & $\beta_{m, 1}$ & $\beta_{m, 2}$ & $\cdots$ & $\beta_{m, m}$ \\
\hline & $w_{1}$ & $w_{2}$ & $\cdots$ & $w_{m}$
\end{tabular}

$$
\begin{array}{c|cccl}
\hat{\alpha}_{1} & \hat{\beta}_{1,1} & \hat{\beta}_{1,2} & \cdots & \hat{\beta}_{1, m} \\
\hat{\alpha}_{2} & \hat{\beta}_{2,1} & \hat{\beta}_{2,2} & \cdots & \hat{\beta}_{2, m} \\
\hat{\alpha}_{3} & \hat{\beta}_{3,1} & \hat{\beta}_{3,2} & \cdots & \hat{\beta}_{3, m} \\
\cdots & \cdots & \cdots & \cdots & \cdots \\
\hat{\alpha}_{m} & \hat{\beta}_{m, 1} & \hat{\beta}_{m, 2} & \cdots & \hat{\beta}_{m, m} \\
\hline & \hat{w}_{1} & \hat{w}_{2} & \cdots & \hat{w}_{m}
\end{array}
$$

Las cuales satisfacen las condiciones siguientes

$$
\begin{aligned}
\alpha_{i} & =\sum_{j=1}^{m} \beta_{i, j}, \\
\hat{\alpha}_{i} & =\sum_{j=1}^{m} \hat{\beta}_{i, j}, \quad i=1,2, \ldots, m
\end{aligned}
$$


Este tipo de métodos se puede aplicar a un sistema Hamiltoniano donde la función Hamiltoniana es separable,

$$
H(\mathbf{q}, \mathbf{p}, t)=K(\mathbf{p})+V(\mathbf{q}, t),
$$

donde $K(\mathbf{p})$ y $V(\mathbf{q}, t)$ son la energía cinética y potencial respectivamente. En este caso el sistema Hamiltoniana toma la forma,

$$
\begin{aligned}
\dot{\mathbf{q}} & =\nabla_{\mathbf{p}} K(\mathbf{p}), \\
\dot{\mathbf{p}} & =-\nabla_{\mathbf{q}} V(\mathbf{q}, t) .
\end{aligned}
$$

Suris, [6] y Sanz-Serna, [5] econtrarón independientemente el resultado siguiente, el cual establece las condiciones para que un método de Ruge-Kutta particionado sea simpléctico cuando es aplicado a un sistema Hamiltoniano separable.

Teorema. Si los coeficientes del método de de Runge-Kutta Particionado satisfacen la relación,

$$
w_{i} \hat{\beta}_{i, j}+\hat{w}_{j} \beta_{i, j}-w_{i} \hat{w}_{j}=0, i, j=1,2, \ldots, m .
$$

Entonces el método RKP es simpléctico cuando es aplicado a un sistema Hamiltoniano con un Hamiltoniano separable.

Demostración. [5, p. 76], [6].

Considere el sistema Hamiltoniano con valores iniciales,

$$
\begin{aligned}
\mathbf{q}^{\prime} & =\mathbf{f}(\mathbf{p}), \quad t_{0} \leq t \leq T, \\
\mathbf{p}^{\prime} & =\mathbf{g}(t, \mathbf{q}) \\
\mathbf{q}\left(t_{0}\right) & =\mathbf{q}_{0}, \mathbf{p}\left(t_{0}\right)=\mathbf{p}_{0} .
\end{aligned}
$$

Aplicando el método de Runge-Kutta particionado a este sistema se obtiene,

$$
\begin{aligned}
\mathbf{q}_{k+1} & =\mathbf{q}_{k}+h \sum_{i=1}^{m} w_{i} \mathbf{f}\left(\mathbf{P}_{i}\right), \\
\mathbf{p}_{k+1} & =\mathbf{p}_{k}+h \sum_{i=1}^{m} \hat{w}_{i} \mathbf{g}\left(t_{k}+\hat{\alpha}_{i} h, \mathbf{Q}_{i}\right),
\end{aligned}
$$

donde

$$
\begin{aligned}
\mathbf{P}_{i} & =\mathbf{p}_{k}+h \sum_{j=1}^{m} \hat{\beta}_{i, j} g\left(t_{k}+\hat{\alpha}_{j} h, \mathbf{Q}_{j}\right), \quad i=1,2, \ldots, m \\
\mathbf{Q}_{i} & =\mathbf{q}_{k}+h \sum_{j=1}^{m} \beta_{i, j} \mathbf{f}\left(\mathbf{P}_{j}\right), \quad i=1,2, \ldots, m .
\end{aligned}
$$

Se han desarrollado y analizado una amplia variedad de métodos de RKP para aproximar la solución de sistemas Hamiltonianos donde la función $H(\mathbf{q}, \mathbf{p}, t)$ es separable. Algunos de estos métodos se encuentran en las referencias, [9], [10] y [11].

\subsection{Métodos de Runge-Kutta-Nyström}

Un caso particular de un sistema Hamiltoniano separable se obtiene de la formulación Hamiltonianan del problema de $N$ cuerpos cuando el sistema es conservativo y además no depende del tiempo, (5). En Hamiltoniano, en este caso, tiene la forma,

$$
H(\mathbf{q}, \mathbf{p})=\frac{1}{2} \mathbf{p}^{t} M^{-1} \mathbf{p}+V(\mathbf{q}) .
$$


Para este tipo de modelos sepuede emplear el método de Runge-Kutta-Nyström. Para derivar el método de parte del Método de Runge-Kutta particionado,

$$
\begin{aligned}
\mathbf{q}_{k+1} & =\mathbf{q}_{k}+h M^{-1} \sum_{i=1}^{m} w_{i} \mathbf{P}_{i} \\
\mathbf{p}_{k+1} & =\mathbf{p}_{k}+h \sum_{i=1}^{m} \hat{w}_{i} \mathbf{f}\left(\mathbf{Q}_{i}\right) \\
\mathbf{P}_{i} & =\mathbf{p}_{k}+h \sum_{j=1}^{m} \hat{\beta}_{i, j} \mathbf{f}\left(\mathbf{Q}_{j}\right) \\
\mathbf{Q}_{i} & =\mathbf{q}_{k}+h \sum_{j=1}^{m} \beta_{i, j} \mathbf{P}_{j}
\end{aligned}
$$

Sustituyendo 20.(c) en 20(a) y 20(d) se obtiene,

$$
\begin{aligned}
\mathbf{q}_{k+1} & =\mathbf{q}_{k}+h^{2} M^{-1} \mathbf{p}_{k}+h^{2} M^{-1} \sum_{i=1}^{m} \hat{\hat{w}}_{i} \mathbf{f}\left(\mathbf{Q}_{i}\right) \\
\mathbf{p}_{k+1} & =\mathbf{p}_{k}+h \sum_{i=1}^{m} \hat{w}_{i} \mathbf{f}\left(\mathbf{Q}_{i}\right) \\
\mathbf{Q}_{i} & =\mathbf{q}_{k}+h \gamma_{i} M^{-1} \mathbf{p}_{k}+h^{2} M^{-1} \sum_{j=1}^{m} \hat{\hat{\beta}}_{i, j} \mathbf{f}\left(\mathbf{Q}_{j}\right)
\end{aligned}
$$

con

$$
\begin{aligned}
\hat{\hat{w}}_{i} & =\sum_{j=1}^{m} w_{j} \beta_{j, i}, \\
\gamma_{i} & =\sum_{j=1}^{m} \beta_{i, j}, y \\
\hat{\hat{\beta}}_{i, j} & =\sum_{k=1}^{m} \beta_{i, k} \hat{\beta}_{k, j} .
\end{aligned}
$$

Esto indica que el método de Runge-Kutta-Nyström se puede realizar con solo una tabla de Butcher con dos arreglos de pesos,

$$
\begin{array}{c|cccl}
\alpha_{1} & \hat{\hat{\beta}}_{1,1} & \hat{\hat{\beta}}_{1,2} & \cdots & \hat{\hat{\beta}}_{1, m} \\
\alpha_{2} & \hat{\hat{\beta}}_{2,1} & \hat{\hat{\beta}}_{2,2} & \cdots & \hat{\hat{\beta}}_{2, m} \\
\alpha_{3} & \hat{\hat{\beta}}_{3,1} & \hat{\hat{\beta}}_{3,2} & \cdots & \hat{\hat{\beta}}_{3, m} \\
\cdots & \cdots & \cdots & \cdots & \cdots \\
\alpha_{m} & \hat{\hat{\beta}}_{m, 1} & \hat{\hat{\beta}}_{m, 2} & \cdots & \hat{\hat{\beta}}_{m, m} \\
\hline & \hat{w}_{1} & \hat{w}_{2} & \cdots & \hat{w}_{m} \\
\hline & \hat{\hat{w}}_{1} & \hat{\hat{w}}_{2} & \cdots & \hat{\hat{w}}_{m} .
\end{array}
$$

\subsection{Experimentos numéricos}

Se aplican algunos de los métodos simplécticos para aproximar la solución al problema de la cuerda vertical. 
4.4.1. La cuerda elástica vertical La cuerda vibrante es un problema que se ha estudiado desde el siglo XVII, [12], [13]. La mayoría de los modelos, de la cuerda vibrante, y los métodos analíticos, para aproximar su solución, asumen desplazamientos pequeños, [14], [15], [16] [17] y [18]. Existen muy pocos modelos para despazamientos grandes, [19], [20]. Se han desarrollado modelos discretos basados en masas y resortes que permiten estudiar la cuerda elástica con desplazamientos arbitrarios, [21], [22], [23]. En la sección de el problema de los $\mathrm{N}$ cuerpos se presento un modelo discreto de una cuerda elástica con un extremo fijo y el otro libre. El modelo produce un sistema Hamiltoniano de ecuaciones diferenciales ordinarias. En esta sección se presentan los resultados de la simulación de la cuerda usando cuatro métodos numéricos diferentes; el método de Euler, [1], el método de Runge-Kutta de orden 3, el método de Störmer-Verlet, [24], y el método de Euler simpléctico, [24].

\begin{tabular}{|l|l|l|}
\hline$L_{c}$ & 1 & Longitud de la cuerda \\
$M_{c}$ & 1 & Masa de la cuerda \\
$N$ & 8 & Número de partículas de la cuerda \\
$m_{i}$ & $M_{c} / N$ & Masa de cada partícula, \\
$l_{i}$ & $L_{c} / N$ & Longitud inicial de cada resorte, \\
$K_{i}$ & 1000 & Cte. de Rigidez del i-ésimo resorte, \\
$g$ & 9,81 & Gravedad \\
$\alpha$ & $1,5625 E-2$ & Impulso inicial al extremo libre \\
\hline
\end{tabular}

Tabla 1: Valores de los parámetros del modelo discreto de la cuerda vertical. i=1.., N

Experimento I. En este experimento los valores de los parámetros del modelo se muestran en la table 1. La longitud del paso de los integradores fue igual a $h=1 / 16384$ y se integro de $t=0$ a $t=12,207$ (200,000 iteraciones). En cada iteración se calculó la energía total del sistema y los resultados se muestran en la figura 6. Todos los métodos empleados funcionan bien para estudiar el problema de la cuerda vertical en tiempos cortos.

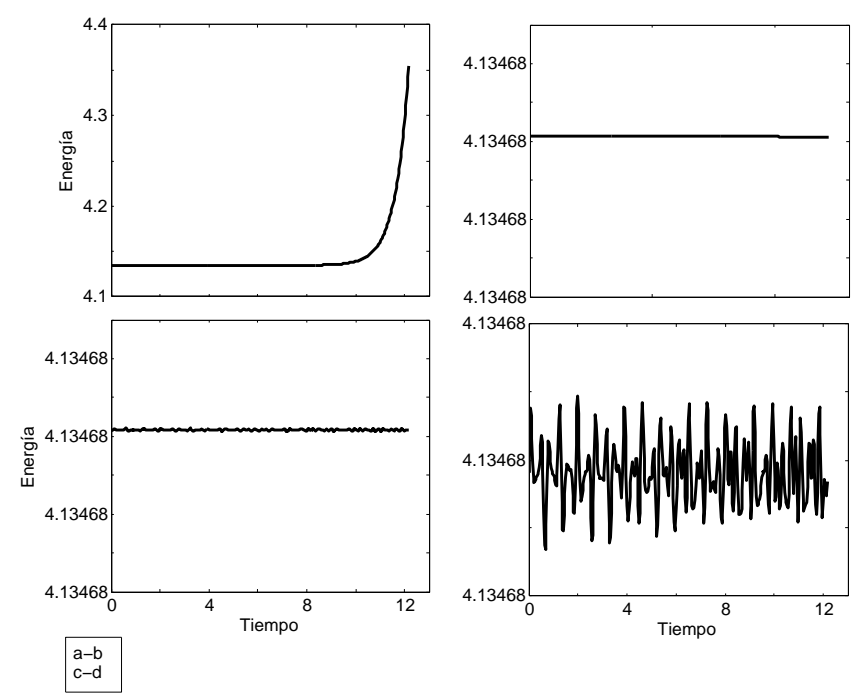

Figura 6: Resultados del experimento I.

El método de Euler empieza a ganar energía exponencialmente a partir de $t=10$. Para integrar un poco más de tiempo, con este método, sin aumentar la energía es necesario disminuir la longitud de paso. Los métodos restantes mantienen la energía constante. Las oscilaciones que presenta el método de Euler simpléctico son menores que $10^{-5}$, figura $6 \mathrm{~d}$.

Experimento II. Los tres mejores métodos del Experimento anterior son empleados para estudiar la cuerda vertical con los mismos parámetros mostrados en la tabla 1. excepto que ahora $N=32$ y $h=1 / 256$. Como el impulso inicial es pequeño los desplazamientos de las partículas también lo serán. 

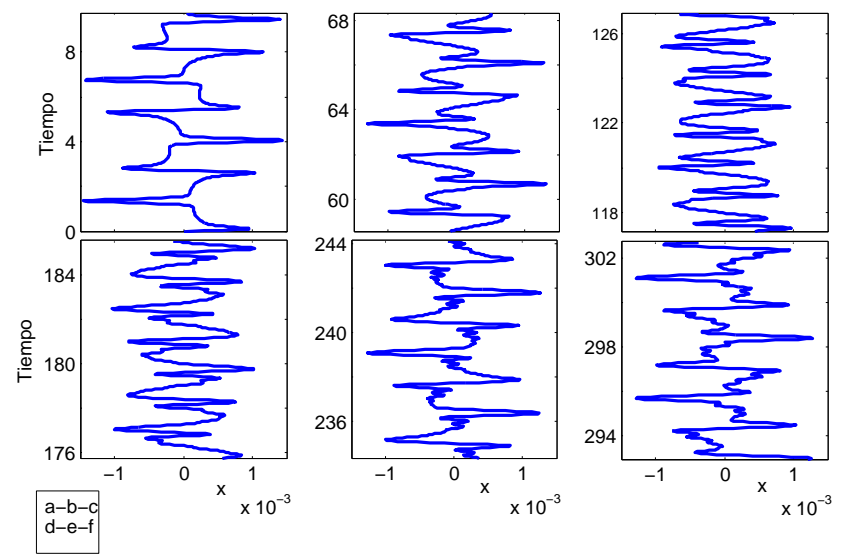

Figura 7: Experimento II. Evolución de la coordenada $x$ del extremo libre de la cuerda. Método de Runge-Kutta
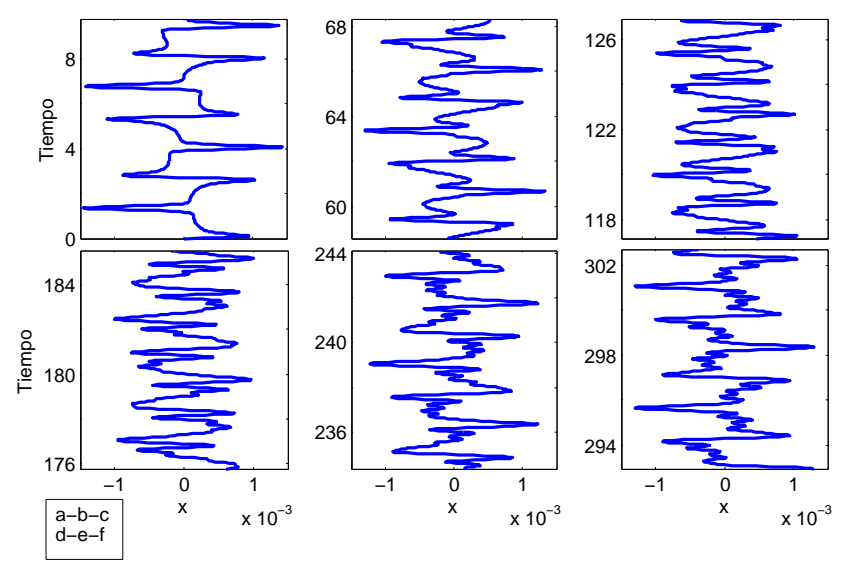

Figura 8: Experimento II. Evolución de la coordenada $x$ del extremo libre de la cuerda. Método de Störmer-Verlet

Los resultados obtenidos al inicio de la simulación, 7 a empatan con la solución analítica del modelo continuo [14]. En la figura 7a-7f se muestran el desplazamiento horizontal $(x)$ del extremo libre de la cuerda vertical, obtenido por el método de Runge-Kutta, en diferentes momentos de su evolución; $10 \mathrm{~s}$ cada 58,5938 s. Toda la evolución se realizó en 100, 0000 iteraciones. Los mismos comentarios se aplican a los resultados con el método de Störmer-Verlet, figura 8 . Los resultados obtenidos con el método de Euler simpléctico son identicos a los de Störmer-Verlet.

Experimento III. En este experimento se analiza el comportamiento de los métodos numéricos empleados con un impulso inicial igual a 2. Los otros paramétros son los mismos que en experimento anterior, la única diferencia es el número de iteraciones, $I t=150,000$.

Los resultados se muestrán en la figuras 911 y 10 . Al inicio de la integración, figuras $9 \mathrm{a}-\mathrm{c}, 11 \mathrm{l}-\mathrm{c}$ y $11 \mathrm{a}-\mathrm{c}$, describen un desplazamiento, del extremo libre, muy similar. Al aumentar el tiempo la evolución los diferentes métodos presenta un desfasamiento entre ellos, figuras $9 \mathrm{~d}-\mathrm{f}, 11 \mathrm{~d}-\mathrm{f}$ y $10 \mathrm{~d}-\mathrm{f}$. Al final de la evolución, figuras 9f, 11f y 10f, cada método muestra una evolución diferente.

Mientras que un método indica que hay chicotazo hacia la derecha otro indica que es hacia la izquierda. Los desplazamientos obtenidos con los métodos simplécticos también están desfasados. Finalmente se grafica la energía del sistema, figura 12. La energía del método de Runge-Kutta decrece exponencialmente, $12 \mathrm{a}$, y se mantiene casi constante para el resto de la evolución. El método de 

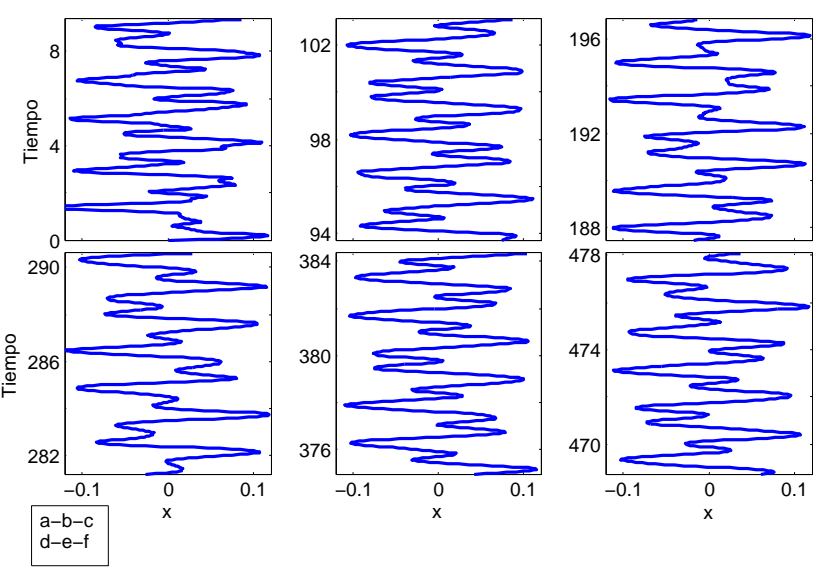

Figura 9: Experimento III. Evolución de la coordenada $x$ del extremo libre de la cuerda. Método de Runge-Kutta
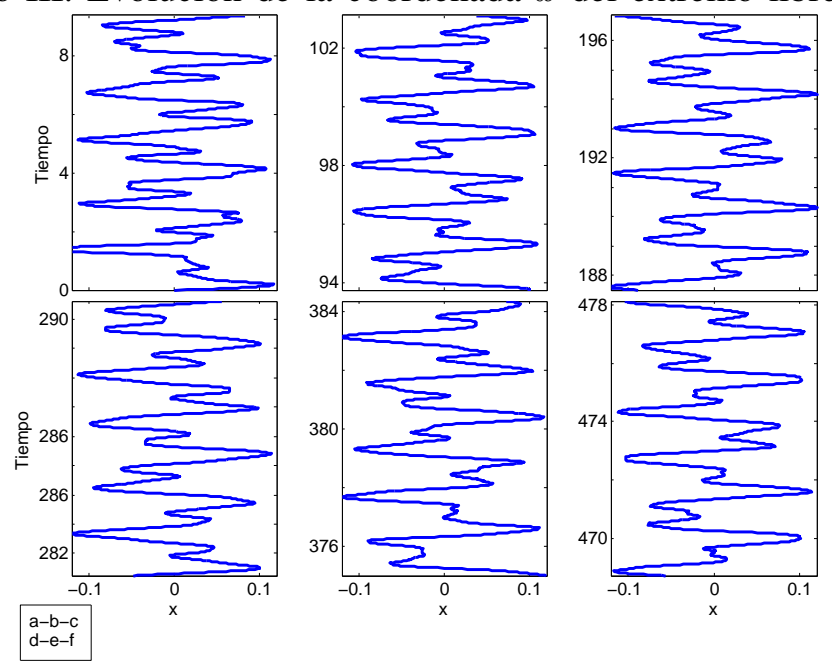

Figura 10: Experimento III. Evolución de la coordenada $x$ del extremo libre de la cuerda. Método de Euler simpléctico
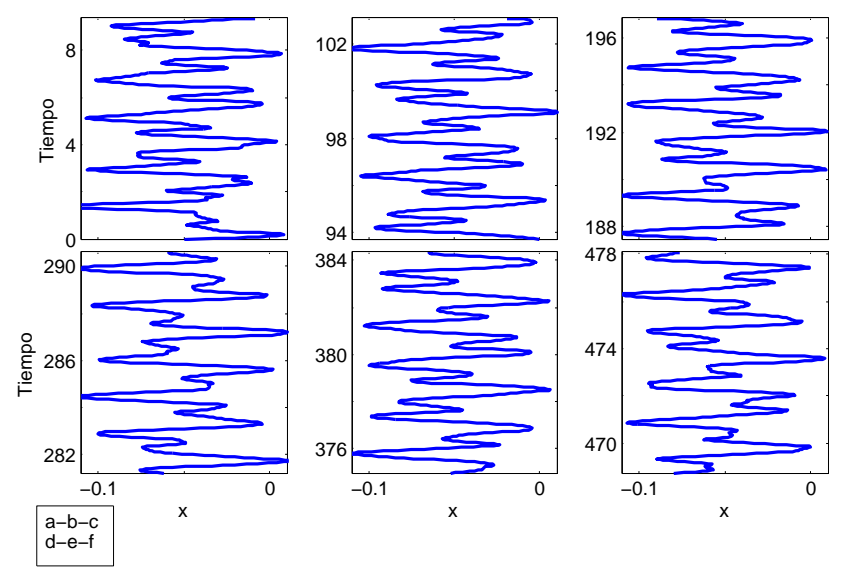

Figura 11: Experimento III. Evolución de la coordenada $x$ del extremo libre de la cuerda. Método de Störmer-Verlet

Störmer-Verlet mantiene la energía constante, figura $12 \mathrm{p}$, y la amplitud de las oscilaciones, alrededor de un valor constante, son menores que las alcanzadas por el método de Euler simpléctico. 


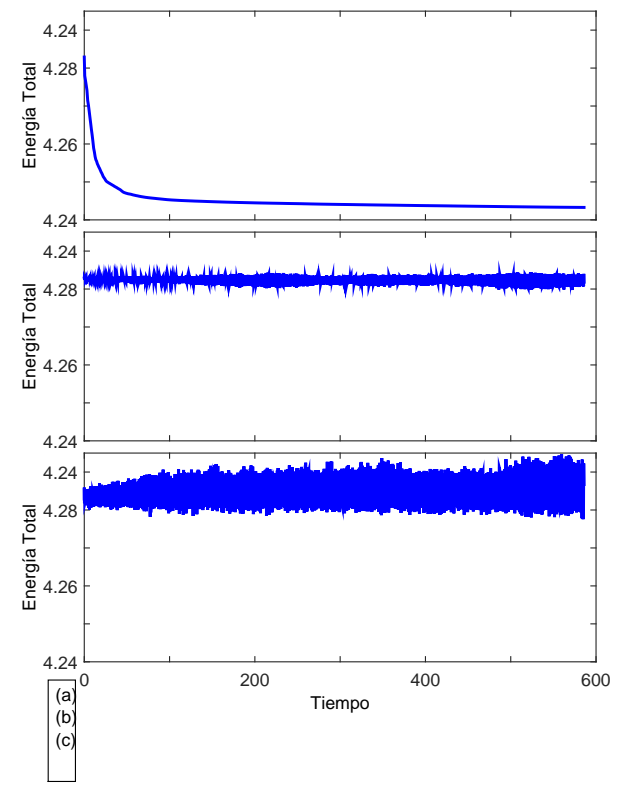

Figura 12: Experimento III. Energía obtenida con los métodos (a) Runge-Kutta, (b)Störmer-Verlet y (c) Euler simpléctico

\section{Conclusiones}

De acuerdo a los experimentos numéricos y a las propiedades de orden y estabilidad de los métodos tipo Runge-Kutta simplécticos son una buena opción para aproximar la solución de sistemas Hamiltonianos. Se empleó el método y algunas de sus variantes; Runge-Kutta particionado y Runge-Kutta-Runge-Kutta-Nyström; para estudiar numéricamente varios sistemas físicos conservativos. En este reporte se presenta un estudio numérico de cuerdas elásticas verticales. Los resultados obtenidos empatan con los reportados por otros investigadores.

\section{Referencias}

[1] K. E. Atkinson, An Introduction to Numerical Analysis. John Wiley and Sons, Inc, 2004.

[2] W. Greiner, Classical Mechanics Systems of Particle and Hamiltonian Dynamics. Springer, 2003.

[3] V. I. Arnold, Mathematical Methods of Classical Mechanics. Springer, 2nd ed., 1989.

[4] E. Hairer, "Important aspects of geometric numerical integration," Journal of Scientific Computing, vol. 25, no. 1-2, pp. 67-81, 2005.

[5] J. M. Sanz-Serna and M. P. Calvo, Numerical Hamiltonian Problems. Chapman and Hall, 1994.

[6] Y. B. Suris, "The canonicity of mapping generated by runge-kutta type methods when integrating the systems $\ddot{x}=\partial u \partial x$," Zh. Vychisl. Mat. i Mat. Fiz. 29(1989)202 - 2011 (en Ruso). Traducción al inglés en: Comput. Math, Math. Phys., no. 29, pp. 138-144, 1991.

[7] F. M. Lasagni, "Canonical runge-kutta methods," Zeitschrift für angewandte Mathematik und Physik ZAMP, vol. 39, no. 6, pp. 952-953, 1988

[8] J. M. Sanz-Serna, "Runge-kutta schemes for hamiltonian systems," BIT Numerical Mathematics, vol. 28, no. 4, pp. 877-883, 1988.

[9] S. Geng, "Symplectic partitioned runge-kutta methods," J. Comput. Math, no. 11, pp. 365-372, 1993.

[10] L. Jay, "Partitioned runge-kutta methods for constrained hamiltonian systems," SIAM Journal on Numerical Analysis, vol. 33 , no. 1,1996

[11] S. Blanes and A. Iserles, "Explicit adaptive symplectic integrators for solving hamiltonian systems," Celest. Mech. Dyn. Astr., no. 114, pp. 297-317, 2012.

[12] J. T. Cannon and S. Dostrovsky, The Evolution of Dynamics Vibration Theory from 1687 to 1742. Springer-Verlag, 1981.

[13] G. N. Watson, A Treatise on the Theory of Bessel Fuynctions. 2nd Ed. Cambridge University Press, 1944. 
[14] H. Bailey, "Motion of hanging chain after the free end is given a initial velocity," Am. J. Physics, vol. 68, no. 8, pp. 764-767, 2000.

[15] J. S. Deschine and B. H. Suits, "The hanging cord with a real tip mass," Eur. J. Phys., no. 29, pp. 1211-1222, 2008.

[16] R. I. Sujith and D. H. Hodges, "Exact solution of free vibrations of a hanging cord with a tip mass," J. Sound Vibr., vol. 32 , no. 12, pp. 359-361, 1995.

[17] C. Y. Wang, "Vibration of a hanging tapered string with or without tip mass," Eur. J. Phys., no. 32, 2011.

[18] D. Yong, "Strings, chains and ropes," SIAM Review, vol. 48, no. 4, pp. 771-781, 2006.

[19] S. S. Antman, “"the equations for large vibration strings"," The American Mathematical Montly, vol. 87, no. 5, pp. 359-370, 1980.

[20] S. S. Antman, Nonlinear Problems in Elasticity. Springer-Verlag, 1995.

[21] D. Greenspan, "Discrete, nonlinear string vibrations," Computer Journal, vol. 13, no. 2, pp. 195-201, 1970.

[22] D. Greenspan, "Computer simulation of transverse string vibrations," BIT, vol. 11, pp. 339-408, 1971.

[23] G. González-Santos and C. Vargas-Jarillo, "A numerical study of a nonlinear hanging string with a tip mass," in Proceeding othe 12th Conference of Computational and Mathematical Methods in Science and Engineering, CMMSE, pp. 1-10, 2012.

[24] E. Hairer, C. Lubich, and G. Wanner, Geometric Numerical Integration - Structure-Preserving Algorithms for Ordinary Differential Equations. Springer-Verlag, New York, 2002.
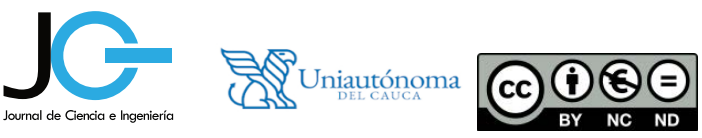